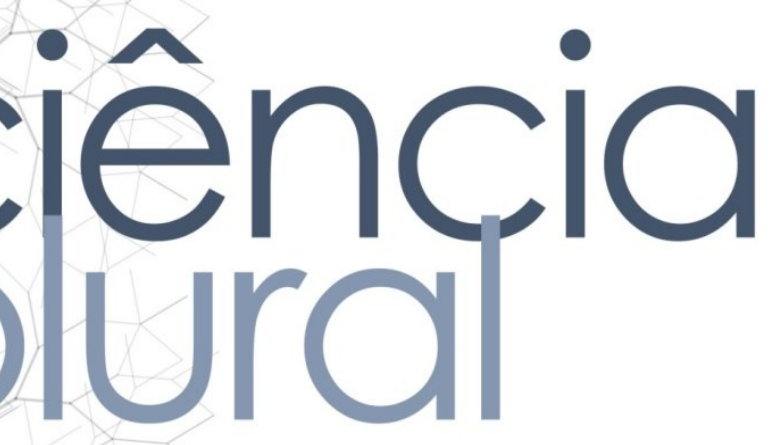

\title{
PERCEPÇÕES DO GRUPO LGBT SOBRE O CÂNCER DE PRÓSTATA: UMA REVISÃO INTEGRATIVA
}

\section{Perceptions from the Igbt group on prostate cancer: an integrating review}

Arlean Salvador da Silva - Graduando do Curso de Enfermagem do Centro Universitário Maurício de Nassau (Uninassau). E-mail: arlleansallvador@gmail.com.

Felyckson Sosttenes Carvalho de Oliveira - Graduando do Curso de Enfermagem do Centro Universitário Maurício de Nassau (Uninassau). E-mail: felycksonsosttenes@gmailcom.

Alexandre Bezerra Silva - Docente do Curso de Ciências Biológica e Médica da Universidade do Estado do Rio Grande do Norte (UERN); Mestre em Saúde da Família (RENASF/UFRN). E-mail: alexandre_enfe@hotmail.com.

Autor responsável pela correspondência:

Felyckson Sosttenes Carvalho de Oliveira. E- mail: felycksonsosttenes@gmailcom. 


\section{RESUMO}

Introdução: Lésbicas, gays, bissexuais, travestis e transexuais LGBT, esse é o grupo protagonista da Política Nacional de Saúde Integral LGBT. Embora com características e pensamentos diferentes, eles vêm reivindicando o livre e pleno acesso às políticas de saúde pública no intuito de prevenir doenças, dentre elas, 0 câncer prostático. Eles estão dentro da área de risco, mas poucos são diagnosticados com esse câncer. Objetivo: Descrever a compreensão do câncer prostático na visão do grupo LGBT. Métodos: Trata-se de um estudo realizado por um levantamento bibliográfico, a partir do método de revisão integrativa de literatura, que tratavam dos seguintes temas: diferenças de gêneros, sexualidade e câncer prostático. Resultados: Os resultados apontam que há dificuldades dos profissionais de saúde relacionados à deficiência no acolhimento, à precariedade do atendimento na Unidade Básica de Saúde (UBS), onde os mesmos são prejudicados por não terem uma assistência diferenciada de acordo com cada gênero e suas peculiaridades. Conclusões: Podese identificar que 0 atendimento nos serviços de saúde às pessoas pertencentes ao grupo LGBT ainda é um desafio. Dentre eles, o fato de que há profissionais da saúde despreparados ao prestar assistência a um paciente transexual, travesti, dentre outros.

Palavras-chave: Câncer de Próstata; Identidade de gênero; Assistência à saúde.

\section{ABSTRACT}

Introduction: LGBT lesbians, gays, bisexuals, transvestites and transsexuals, this is the main group of the National LGBT Comprehensive Health Policy. Although with different characteristics and thoughts, they have been claiming the free and full access to public health policies in order to prevent diseases, among them, prostate cancer. They are within the risk area, but few are diagnosed with this cancer. Objective: To describe the understanding of prostate cancer in the view of the LGBT group. Methods: This is a study carried out by a bibliographical survey, based on the integrative literature review method, which dealt with the following themes: gender differences, sexuality and prostate cancer. Results: The results indicate that there are difficulties of the health professionals related to the deficiency in the reception, to the precariousness of the care in the Basic Health Unit (UBS), where they are harmed by not having a differentiated care according to each gender and its peculiarities. Conclusions: It can be identified that the care in the health services to the people belonging to the LGBT group is still a challenge. Among them, the fact that health professionals are unprepared when assisting a transsexual patient, transvestite, among others.

Keywords: Prostate cancer; Gender identity; Health care. 


\section{INTRODUÇÃO}

Observa-se um crescimento do movimento de lésbicas, gays, bissexuais, travestis e transexuais (LGBT). Entretanto, há evidências de fatores relacionados à organização dos serviços, à postura de profissionais, ao estigma e ao constrangimento vivenciado pela população LGBT, que comprometem a materialização dos princípios e diretrizes do Sistema Único de Saúde-SUS1.

Nesse sentido, se existem avanços, especialmente no que se referem a travestis, transexuais e transgêneros, representados, principalmente, pela formalização de programas governamentais e pelo incremento do debate público, há também desafios estando um deles no setor saúde².

A população LGBT vem se organizando com o intuito de reivindicar os direitos de ir e vir, a livre expressão da orientação sexual, como também o livre acesso às políticas de saúde pública, no intuito de amenizar os casos de agressões e mortes causadas por patologias, considerando o direito à saúde garantido no art. 196 da Constituição Federal.

Estudos mostram que quando se remete à saúde, logo entende-se voltada para a categoria heterossexual, limitando suas atuações efetivas junto a pacientes LGBT. Dessa forma, esse entendimento irá interferir negativamente nas formas de cuidado que os usuários LGBT recebem em determinados serviços de saúde, dificultando também o acesso desse público aos serviços de saúde tornando esse acesso problemático aos serviços de saúde a essa população mais vulnerável em adquirir doenças ${ }^{3}$.

Diante desse cenário, lésbicas, gays, bissexuais, travestis e transexuais, esse é o grupo protagonista da Política Nacional de Saúde Integral LGBT. Embora com identidades, características e pensamentos diferentes, eles vêm reivindicando o livre e pleno acesso às políticas de saúde pública no intuito de prevenir doenças, dentre elas, a neoplasia prostática. Eles estão dentro da área de risco, mas poucos são diagnosticados com esse câncer ${ }^{3}$.

A próstata é uma glândula exócrina, anexado ao aparelho genital masculino que está situada abaixo da bexiga e envolve a parte inicial da uretra, o tamanho da próstata varia com a idade. Sua função é armazenar e secretar fluído alcalino que protege e nutre os espermatozoides e que constitui parte do sêmen 4 . Essa doença é a segunda mais incidente no mundo e a quinta causa de mortalidade por tumores malignos. No Brasil de acordo com o Instituto Nacional de Câncer (INCA) temos a estimativa de novos casos para os próximos anos de 61.200 mil pessoas acometidas com esse tipo de câncer, gerando um agravamento para saúde pública 5 . 


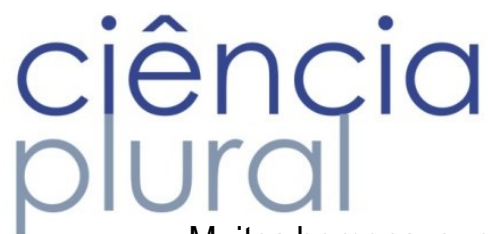

Muitos homens, que compõem o grupo LGBT, também podem desenvolver a doença, sem apresentar quaisquer sintomas. Isso faz com que a incidência aumente, deixando os homens mais vulneráveis à essa patologia. Vale ressaltar o grupo de mulheres transexuais, embora pertencente ao gênero feminino, permanecem com a próstata, desse modo também merecendo atenção ${ }^{6}$.

0 câncer prostático se caracteriza por ser assintomático e sintomático. Na fase inicial demonstra evolução silenciosa de forma que se assemelhe ao desenvolvimento benigno da próstata, dificuldade em urinar, necessidade em urinar, febre e outros sintomas. Durante a fase avançada pode apresentar sintomas como: dor óssea, urinária e insuficiência renal em casos de infecção generalizada ${ }^{7}$.

Percebe-se que na área da saúde ainda há desrespeito com a população LGBT, mesmo após a implantação da Política Nacional de Saúde LGBT. Há uma fragilização no atendimento por parte dos profissionais da saúde de pessoas que a identidade de gênero difere do sexo biológico, o qual contribui para a violação dos direitos humanos básicos como, por exemplo, a violação do direito à saúde, por não ter um atendimento humanizado ${ }^{8}$. Nessa perspectiva, o objetivo desse estudo é descrever a compreensão do câncer prostático na visão do grupo LGBT.

\section{METODOLOGIA}

Este estudo caracteriza-se como uma revisão integrativa, com coletas de dados realizadas a partir de fontes secundárias, por meio de levantamento bibliográfico. A revisão integrativa é um método de pesquisa que permite a sintese de múltiplos estudos publicados e possibilita conclusões gerais a respeito de uma partícula área de estudo ${ }^{9}$. O levantamento bibliográfico foi realizado entre maio e julho de 2018.

Para a seleção da amostra foram utilizados os seguintes critérios de inclusão: artigos que abordassem a compreensão do câncer prostático na população LBGT, assistência de saúde para população LGBT e publicações que estivessem entre 2007 e 2017. Foram utilizadas as seguintes chamadas: neoplasia prostática, câncer prostático, identidade de gênero e assistência à saúde. Para compor o corpus da pesquisa, buscou-se artigos disponiveis online nas seguintes bases de dados: Literatura Latino-americana e do Caribe em Ciências Sociais e da Saúde (LILACS) e Scientific Eletronic Library (SCIELO), incluídos na Biblioteca Virtual de Saúde (BVS). Foram utilizados apenas artigos completos publicados em português.

Os artigos selecionados foram os que tratavam do câncer prostático, acolhimento, população LGBT, identidade de gênero sua percepção diante ao câncer prostático, verificando que todos os artigos apresentaram objetivos claros a respeito do tema aqui escolhido. Foram encontrados 45 trabalhos, de acordo com o título e objetivo do nosso estudo. Após uma primeira leitura, foram excluídos os trabalhos que tinham como foco sexualidade e doenças sexualmente transmissíveis (DSTs), restando 12 artigos 


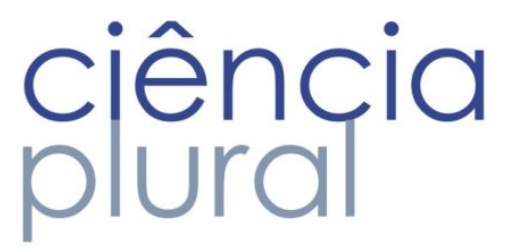

selecionados. Alguns artigos selecionados foram publicados nas seguintes revistas: Revista Latino Americano, Revista Conbracis e Revista Gaúcha de Enfermagem.

Desta forma, os artigos selecionados foram lidos na íntegra. É visto que ainda há poucos trabalhos que tenham uma discussão sobre a compreensão da população LGBT frente ao câncer de próstata. São raros os trabalhos que abordam esse tema ao grupo LGBT e, mesmo diante dos trabalhos encontrados, há poucas informações sobre a saúde/doença (câncer de próstata) e as particularidades de cada gênero e identidade que compõem o grupo LGBT.

\section{RESULTADOS E DISCUSSÃO}

\section{Rastreamentos do câncer prostático}

Os dois principais norteadores do rastreamento do câncer prostático são: Antígeno prostático especifico (PSA), e o Toque Digital Retal. O PSA é uma glicoproteína originária na próstata, e o seu nível elevado na corrente sanguínea é considerado um importante marcador biológico para algumas doenças da próstata, entre elas, o câncer. $O$ antígeno prostático que é identificado pela glicoproteína é produzido pelas células epiteliais da próstata e não especificamente pela célula cancerosa, podendo também estar alterado em outras patologias e resultar na realização de biópsia desnecessária ${ }^{6}$.

O toque digital retal, mais conhecido por toque retal, é uma medida preventiva de baixo custo, no entanto é um procedimento essencial do exame físico realizado com a população que procura o serviço de saúde para diagnóstico precoce do câncer de próstata, o qual tem o objetivo na identificação de anormalidades, abaulamentos e alterações da consistência deste órgão chamado próstata, reduzir a incidência da doença tardia interferindo nas taxas de mortalidade. Vale ressaltar que o objetivo desse exame é propor ao examinador a possibilidade de conhecer as dimensões, o formato e os limites do órgão ${ }^{10}$.

O câncer de próstata pode ser curável se for detectado previamente, mesmo que o tratamento hormonal seja utilizado pela população LGBT, o estrógeno é um hormônio feminino que causa alterações no corpo, como as alterações das mamas, entre outros. O público LGBT ao fazer uso deste hormônio tem mudanças fisiológicas de um corpo masculino para um feminino. Dado o tratamento hormonal, a administração deste hormônio muda as alterações do corpo, mas mesmo assim é necessária a realização do exame retal, pois o aumento da próstata vai ocorrer de acordo com a idade mesmo com o uso do hormônio ou não, ou seja, a população LGBT deve sim realizar o rastreamento da neoplasia prostática ${ }^{11}$. 


\section{Aspecto do Processo Saúde/Doença da população LGBT}

A discussão sobre o processo saúde/doença do grupo LGBT esteve presente em alguns artigos, sendo definida de várias maneiras, conforme veremos a partir dos trabalhos de Albuquerque ${ }^{12} \mathrm{e}$ Cavalcanti13.

Ao abordarem a problemática da saúde no grupo LGBT, o primeiro ponto destacado relaciona-se ao direito à saúde, que é conceituado como categoria de direito subjetivo público, visto que todos devem ter 0 livre acesso a assistência de saúde, no qual precisa-se entender sobre os fatores de risco que venham desencadear problemas para sua saúde ${ }^{12}$.

Essa problemática do acesso aos serviços de saúde torna essa população mais suscetível a adquirir doenças mais prevalentes a esse público, tais como: problemas de cunho psicológicos, Síndrome da Imunodeficiência Adquirida (AIDS), câncer de colo do útero, câncer de mama e câncer de próstata. 0 que justifica a prevalência dessas doenças nesse público é justamente a falta de um acompanhamento e conhecimento sobre o processo do cuidado com seu corpo para que se faça prevenção ${ }^{13}$.

O que justifica a prevalência dessa doença nesse público é justamente a falta de um acompanhamento de rotina para prevenção da mesma, visto que há vários fatores que propiciam uma lacuna no atendimento desse público como, por exemplo, o respeito ao uso do nome social, tornando cada vez menor a compreensão da população frente ao câncer. A lacuna no atendimento é um fator primordial para o aumento de casos com câncer prostático, sabemos que o grupo LGBT está dentro da área de risco, mesmo os que têm uma identidade de gênero diferente ao sexo biológico, isso não vai impedir que os mesmos não pudessem adquirir a doença ${ }^{14}$.

Ao mesmo tempo que surgem as buscas pelos direitos de cidadania, fica evidente que no Brasil, ainda é escasso o conhecimento das necessidades em saúde da população LGBT, conhecimento esse que se evidencia como primordial para fomentar o desenvolvimento das políticas de saúde direcionadas à categoria ${ }^{15}$.

A partir desse cenário, é importante ressaltar e compreender que, por mais necessário que se apresente ouvir a voz da população LGBT sobre suas necessidades e perspectivas, objetivo central em nosso trabalho, os artigos selecionados não continham, de forma direta, a visão da população LGBT em relação ao câncer de próstata. 


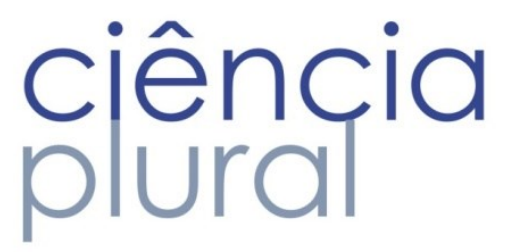

\section{CONCLUSÕES}

Diante do exposto, pode-se identificar que 0 atendimento nos serviços de saúde às pessoas pertencentes ao grupo LGBT ainda é um desafio. Dentre eles, o fato de que há profissionais da saúde despreparados ao prestar assistência a um paciente transexual, travesti, dentre outros. Os estudos apontam que a identidade de gênero e a orientação sexual não são tratadas com o grau de relevância merecido, em consequência, os profissionais da saúde deixam-se levar pelo déficit da não assistência da população LGBT, fazendo com que estes passem a ter um desconhecimento das redes de atenção a sua saúde, das políticas, aumentando a sua restrição frente ao serviço de saúde.

Com isso, nota-se que o nível de compreensão se torna cada vez menor quanto ao câncer de próstata, se esse vínculo entre saúde e cuidado não for associado. Essa compreensão iria facilitar o conhecimento do grupo LGBT sobre meios de prevenção, diagnóstico e tratamento.

É possível considerar que, o baixo conhecimento sobre essa temática é um fator crucial para que a população fique mais restrita quanto a busca pela promoção da saúde e prevenção de doença. Acredita-se que quanto maior for o conhecimento, melhor será a compreensão dos mesmos, quando se refere ao processo saúde/doença e ao cuidado com seu corpo, tendo em vista que todos esses fatores irão potencializar igualdade de direitos que contribuam para construção de metas que visem a melhoria da qualidade de vida da população LGBT. Nessa direção, em pesquisas futuras, é de fundamental importância trazer à tona as vozes da população LGBT frente a seus embates e necessidades.

\section{REFERÊNCIAS}

1. Freire EC, Araujo FCA, Sousa AD, Marques D. A clínica em movimento na saúde de TTTS: caminho para materialização do SUS entre travestis, transsexuais e transgêneros.Saúde em Debate. Rio de Janeiro. v. 37. n. 98, p. 477-484, jul/set 2013. Disponivel em: < http://www.scielo.br/scielo.php?pid=S010311042013000300011\&script=sci_abstract\&tlng=pt>

2. Facchini R. Entre compassos e descompassos: um olhar para o "campo" e para a "arena" do movimento LGBT brasileiro. Bagoas, São Paulo. n. 4, p.131-158, 2009. Disponivel em: http://www.clam.org.br/bibliotecadigital/uploads/publicacoes/entre\%20compassos\%20e\%20descompa ssos.pdf

3. Borges CA, Souza M. Saúde das travestis: um desafio para a enfermagem. UNIFRA. Rio grande do Sul. 2012. 


\section{ciência \\ plural}

http://www.scirp.org/(S(i43dyn45teexjx455q|t3d2q))/reference/ReferencesPapers.aspx?ReferencelD= 1240325>.

4. Bertoldo AS, Pasquini ZV. Câncer de próstata: um desafio para a saúde do homem. Ver enfermagem UNISA. N.11(2), 2010.

Disponivel em: https://www.unisa.br/graduacao/biologicas/enfer/revista/arquivios/2012-2-15.pdf >.

5. Brasil. Ministério da Saúde. Instituto Nacional de Câncer. Programa Nacional de controle do câncer da próstata: documento de consenso. Rio de Janeiro (RJ): INCA, 2002. Disponível em: $<$ <ttp://bvsms.saude.gov.br/bvs/publicacoes/cancer_da_prostata.pdf>.

6. Amorim VMSL, Barros MBA, Cesar CLG, Galdbaun M, Garandina L, Alves MCGP. Fatores associados à realização dos exames de rastreamento para o câncer da próstata. Caderno Saúde Pública do Rio de Janeiro. n.27(2), p.347-356, fev., 2011. Disponível em: <http://www.scielo.br/pdf/csp/v27n2/16.pdf>.

7. Bacelar júnior AJ, Menezes CS, Barbosa CA, Freitas GBS, Silva GG, Vaz JPS, et al. Câncer de próstata: métodos de diagnóstico, prevenção e tratamento. Faculdade Presidente Antônio Carlos. Vol.10, n.3, p.40-46, mar - mai., 2015. Disponível em: $<$ http://www.mastereditora.com.br/periodico/20150501_174533.pdf>.

8. Mello L, Perilo M, Braz CA, Pedrosa C. Políticas de saúde para lésbicas, gays, bissexuais, travestis e transexuais no Brasil: em busca de universalidade, integralidade e equidade. Revista Latino Americana. n.9, p.7-28, 2011. Disponivel em: <http://www.scielo.br/pdf/sess/n9/02.pdf >.

9. Mendes KDS, Silveira RCCP, Galvão CM. Revisão integrativa: Método de Pesquisa para a Incorporação de Evidências na Saúde e na Enfermagem. Texto contexto - enferma. Florianópolis, v. 17, n. 4, dez. 2008. Disponível em: <http://dx.doi.org/10.1590/S0104-07072008000400018>.

10. Oliveira PSD, Alves AM, Rocha RMP, Barbosa HA. Percepção dos homens sobre o exame toque retal. Enfermagem residente da família da UFMG. Revista Enferm. UFPE online, Recife, 9(5): 7760-5, maio, 2015.

Disponível

em: $<$ http://bases.bireme.br/cgibin/wxislind.exe/iah/online/?lsisScript=iah/iah.xis\&src=google\&base=BDEN F\&lang=p\&nextAction=Ink\&exprSearch=28253\&indexSearch $=\mid \mathrm{D}>$.

11. Rohden F. O homem é mesmo a sua testosterona: Promoção da andropausa e representações sobre sexualidade e envelhecimento no cenário brasileiro. Universidade Federal do Rio Grande do Sul-Brasil. Porto alegre, ano 17, $\mathrm{n}$ 35, p.161-196, jan/jun.2011. Disponível em: $<$ http://www.scielo.br/pdf/ha/v17n35/v17n35a06.pdf>.

12. Albuquerque GA, Garcia CL, Alves MJH, Queiroz CMHT, Adami F. Homossexualidade e o direito à saúde: um desafio para as políticas públicas da saúde no Brasil. Saúde em Debate, Rio de Janeiro, v. 37, n. 98, p. 516-24, 2013. Disponível em: <http://www.scielo.br/pdf/sdeb/v37n98/a15v37n98.pdf>. 
13. Cavalcanti AC, Nascimento LC, Medeiros HAH, Nunes ASR, Barreto AJR. Acolhimento nos serviços de saúde a população LGBT: uma revisão integrativa. Universidade Federal de Campina Grande - Campus Cuité - $\quad$ UFCG. Disponivel em: <http://www.editorarealize.com.br/revistas/conbracis/trabalhos/TRABALHO_EV055_MD1_SA4_ID365 _30052016231804.pdf >.

14. Souza JC, Mallmann DG, Neto NMG, Freitas NO, Vasconcelos EMR, Araújo EC. Promoção da saúde da mulher lésbica: cuidados de enfermagem. Revista Gaúcha Enferm. v.4, n.35, p.108-13, 2014. Disponível em: <http://www.scielo.br/pdf/rgenf/v35n4/pt_1983-1447-rgenf-35-04-00108.pdf >.

15. Santos EC Calvetti PU, Rocha KB, Moura A, Barbosa LH, Hermel J. Percepção de usuários gays, lésbicas, bissexuais e transgêneros, transsexuais e travestis do sistema único de saúde. Revista interamericana de psicologia/interamerican journal of psychology. 2010, 44(2):235-245. 